\title{
Article \\ Computational Modeling of Ultrasound C-Scan Imaging Using Transmitted Signal Peak Density
}

\author{
Koushik Paul ${ }^{1} \oplus$, Jeremy Stromer ${ }^{2}$, Samuel Razmi ${ }^{3}$, Barbara A. Pockaj ${ }^{4}$, Leila Ladani ${ }^{1}\left(\mathbb{D}\right.$ and Jafar Razmi ${ }^{5, *}$ (1) \\ 1 School for Engineering of Matter, Transport and Energy, Ira A. Fulton Schools of Engineering, \\ Arizona State University, Tempe, AZ 85281, USA; kpaul5@asu.edu (K.P.); ladani@asu.edu (L.L.) \\ 2 Survivability Engineering Branch, US Army Engineer Research and Development Center, \\ Vicksburg, MS 39180, USA; jeremy.d.stromer@erdc.dren.mil \\ Texas A\&M College of Medicine, Houston, TX 77807, USA; samrazmi@tamu.edu \\ 4 Mayo Clinic in Arizona, Surgery, Phoenix, AZ 85054, USA; pockaj.barbara@mayo.edu \\ 5 School of Sustainable Engineering and the Built Environment, Ira A. Fulton Schools of Engineering, \\ Arizona State University, Tempe, AZ 85281, USA \\ * Correspondence: jafar.razmi@asu.edu; Tel.: +1-(480)-965-2543
}

Citation: Paul, K.; Stromer, J.; Razmi, S.; Pockaj, B.A.; Ladani, L.; Razmi, J Computational Modeling of Ultrasound C-Scan Imaging Using Transmitted Signal Peak Density. Appl. Sci. 2021, 11, 4924. https:// doi.org/10.3390/app11114924

Academic Editor: Filippo Berto

Received: 30 March 2021

Accepted: 24 May 2021

Published: 27 May 2021

Publisher's Note: MDPI stays neutral with regard to jurisdictional claims in published maps and institutional affiliations.

Copyright: (c) 2021 by the authors. Licensee MDPI, Basel, Switzerland. This article is an open access article distributed under the terms and conditions of the Creative Commons Attribution (CC BY) license (https:// creativecommons.org/licenses/by/ $4.0 /)$.

\begin{abstract}
Ultrasound measurement is a relatively inexpensive and commonly used imaging tool in the health sector. The through-transmission process of ultrasound measurement has been extensively evaluated for detecting abnormalities in tissue pathology. Compared to standard imaging parameters such as amplitude and time of flight, quantitative ultrasound parameters in the frequency domain can provide additional details regarding tissue microstructures. In this study, pressure magnitude or amplitude variation in the frequency spectrum of the received signal was evaluated as a potential imaging technique using the spectral peak density parameter. Computational C-scan imaging analysis was developed through a finite element model. The magnitude variation in the received signal showed different patterns while interacting with and without inclusions. Images were reconstructed based on peak density values that varied with the presence of solid structure. The computational results were verified with the experimental C-scan imaging results from the literature. It was found that magnitude variation can be an effective parameter for C-scan imaging of thin structures. The feasibility of the study was further extended to identify the structure's relative position along with the sample depth during C-scan imaging. While moving the structure in the direction of the sample depth, the pressure magnitude variation strongly followed a second-degree polynomial trend.
\end{abstract}

Keywords: acoustics; C-scan imaging; ultrasound analysis; finite element model; pitch-catch; frequency spectrum; pressure variation

\section{Introduction}

Ultrasound imaging is an effective imaging technique in the industrial and health sector as it is nondestructive, nonionizing, and relatively inexpensive [1]. It has the potential of having several centimeters of penetration depth, depending on the frequency range used [2]. Three types of scanning modes are available in ultrasound imaging, which are A-scan, B-scan, and C-scan. The A-scan mode works one-dimensionally, only providing amplitude data of the returning echoes from various reflectors situated along the wave propagation direction. On the other hand, the B-scan and C-scan modes provide a two-dimensional image of the plane parallel and perpendicular to the wave direction, respectively [3].

The image quality in the C-scan mode highly depends on the lateral resolution of the ultrasound, as the imaging plane is perpendicular to the wave direction. Therefore, the element size used in the ultrasound transducer, as well as the focusing ability of the transducer crystal, plays heavily in determining the image quality in C-scan mode. Additionally, the frequency level also plays an important role in the image resolution, as the high-frequency ultrasound beam diverges slowly, which makes the beam diameter smaller 
in the far zone [4]. Furthermore, during image reconstruction, parameter measurement inaccuracies can result in low image quality (e.g., poor edge detection), for which fuzzy preprocessing procedures are applied [5,6]. Both pulse-echo and pitch-catch techniques were evaluated to produce C-scan images in ultrasonic measurements [7]. Pitch-catch methods are mostly used in industrial applications, whereas pulse-echo techniques have been adopted in medical applications [7-16].

In the industrial sector, C-scan images can provide very high-resolution subsurface images that can evaluate defect size, delamination, and welding quality $[9,10,15]$. Although in the medical sector B-scan imaging is widely used, the imaging process only uses the RF signal envelope [1]. It fails to provide quantitative measurement related to biological and structural attributes for characterization [17-20]. On the other hand, a C-scan image can utilize the quantitative ultrasound (QUS) parameters that were used for monitoring cell decay, analyzing cardiac irregularities, and characterizing tumors $[1,21]$. Thus, by utilizing QUS, a C-scan image can potentially characterize various tissue properties. For example, in the ophthalmology field, by measuring the ultrasonic backscatter properties, C-scan imaging was used to evaluate eye tissue structures [13]. Various ultrasound parameters of the C-scan method have been evaluated so far. Standard parameters such as amplitude, time of flight, and quantitative parameters such as attenuation and spectral intensity have been utilized in C-scan imaging [7,9,22]. Some nonconventional parameters have also been investigated to improve the image quality further in terms of precise detection [22]. One of those parameters is spectral peak density. Coined by Doyle et al., peak density represents the total number of peaks and valleys present in a frequency spectrum. Therefore, it represents the magnitude variation of the received signal [21]. Peak density was found to be very sensitive towards the microstructural variation in soft materials [23]. This parameter was evaluated by Stromer et al., where it was compared against standard ultrasound parameters [22]. Peak density showed similar and, in some cases, better performance compared to the other parameters; however, the quality of the image was dependent on the application through an intuitive threshold magnitude criterion [22].

Acoustic scattering also plays an important role in evaluating ultrasound parameters during wave propagation in the forward direction [24]. Depending on the size of the structure and ultrasound wavelength, three different levels of scattering can occur. Stromer et al. performed C-scan imaging using specular scattering where the solid structure size was much larger compared to the ultrasound wavelength. In that case, the wave did not scatter in all directions, but rather, some portions of the wave traveled directly through the structure, while the rest of it went back to the source. The amount and direction of the transmitted wave depend on the impedance mismatch between the two mediums and the incident wave direction, respectively.

In the experimental C-scan imaging conducted by Stromer et al., the pitch-catch method was conducted, where two high-frequency transducers of $25 \mathrm{MHz}$ were used [22]. The bandwidth frequency range of the transducer was $22-41 \mathrm{MHz}$. The received signal was converted to a frequency spectrum, and the magnitude variation of that signal was measured in terms of peak density. Based on the peak density value at different locations of the phantom, the C-scan image was reconstructed. For high-frequency imaging, soft pepper flakes and hard pepper seeds were inserted for detection inside the gelatin-based tissue phantom. Although peak density successfully imaged the pepper seed, because it was a hard scatterer, it could not identify the soft pepper flake's edge [22]. Therefore, to establish the peak density parameter as an effective through-transmission imaging tool, this limitation needs to be properly addressed and eradicated.

In this study, a computational model of C-scan imaging was developed. COMSOL Multiphysics was used to perform the finite element analysis. A 2D model was developed to keep the analysis computationally inexpensive. The analysis was conducted in different cross-sections obtained from the original 3D model. The first objective of this study was to mimic the experimental analysis of Stromer et al. by creating a computational model similar to the experimental C-scan imaging setup. Ultrasound propagation was conducted 
in a parametric study to mimic the imaging technique. A general Gaussian function was introduced in the incident pressure to imitate the pressure distribution in transducer bandwidth. After validating the simulation result by the experimental result obtained by Stromer et al., the computational model was further utilized to detect the relative position of structure along the acoustic propagation path in the same model.

\section{Finite Element Analysis (FEA) Simulation}

\subsection{Model Description}

The first objective of this study was to mimic the experimental analysis of Stromer et al. [22]. Therefore, the simulation model geometry was created by imitating the experimental sample. A gelatin phantom containing soft pepper flakes was modeled for the simulation. The pepper flake was modeled as a thin cylinder of $3 \mathrm{~mm}$ diameter and $50 \mu \mathrm{m}$ height. The gelatin phantom was created as a cube of $5 \mathrm{~mm}$ in length at each side. The 3D model geometry is shown in Figure 1. The center pepper flake was placed at $0.2 \mathrm{~mm}$ along the negative $Y$-axis from the phantom center.

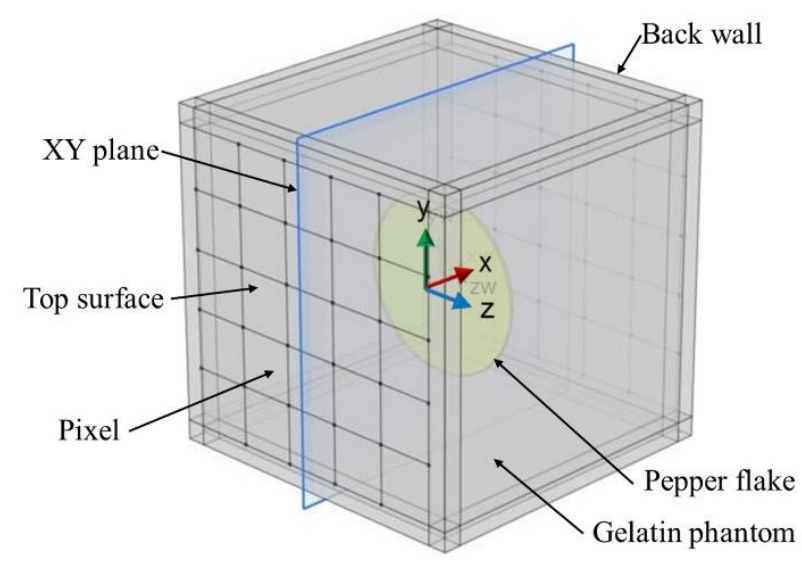

Figure 1. Three-dimensional (3D) model geometry of the gelatin phantom containing the pepper flake.

In the study of Stromer et al., two single-element transducers were used for the C-scan imaging, where each transducer crystal diameter was $2 \mathrm{~mm}$ [22]. A raster scan pattern was used while selecting the step size as half of the crystal diameter, resulting in an image resolution of $1 \mathrm{~mm} /$ pixel. Therefore, in this computational model, the top surface of the $3 \mathrm{D}$ model was divided into 25 pixels, with each pixel area having a dimension of $1 \mathrm{~mm}$ on each side. Thus, the image resolution became $1 \mathrm{~mm} /$ pixel. Similar to the experimental study, ultrasound imaging was conducted in a through-transmission or pitch-catch method. The signal was sent from the top surface and received at the bottom surface (back wall). After receiving the signal, the peak density of the frequency spectrum was measured. The peak density value was then used as the pixel value for the C-scan image reconstruction.

\subsection{Model Simplification}

In the case of the 3D model, because of the high frequency, the number of mesh elements was too high. To keep the model computationally inexpensive, the 3D model shown in Figure 1 was divided into $25 \mathrm{XY}$ cross-sectional planes along the Z-axis. The peak density result (Section 2.4) was found very consistent from one cross-section to another. Thus, it was approximated that even with an increasing number of cross-sectional planes (e.g., 50 or 100 or more), the change in the average peak density value for each pixel would be very insignificant.

The distance between each plane was kept as $0.25 \mathrm{~mm}$. Figure 2a shows 5 of those 25 cross-sectional planes from the middle of the 3D geometry. During model simplification, the pixel area was converted into lines. Therefore, instead of conducting simulation in the 3D model, the simulation was performed on the simplified 2D model. Finally, the 
results from all the 2D models were accumulated to achieve the 3D model result. In the 2D model shown in Figure 2b, the X-direction indicates the depth of the sample, as well as the ultrasound propagation direction. The Y-direction indicates the width of the phantom. The gelatin sample was surrounded by a perfectly matched layer to ensure uninterrupted wave propagation (Figure 2b). The 2D geometry was divided into five sectors. The sectors were numbered numerically from top to bottom (Figure $2 b$ ). In the 2D model, each sector area acted as a pixel volume (voxel) from the 3D model. The ultrasound wave was sent through each sector in the positive X-direction in a parametric study. For each frequency step, the ultrasound wave pressure was measured at the back wall of each sector. Peak density was calculated from the signal received from the back wall line, as shown in Figure $2 \mathrm{~b}$. Because each pixel area from the 3D model was converted into five lines (five 2D models), the average peak density value from those five $2 \mathrm{D}$ models was considered the corresponding pixel value.

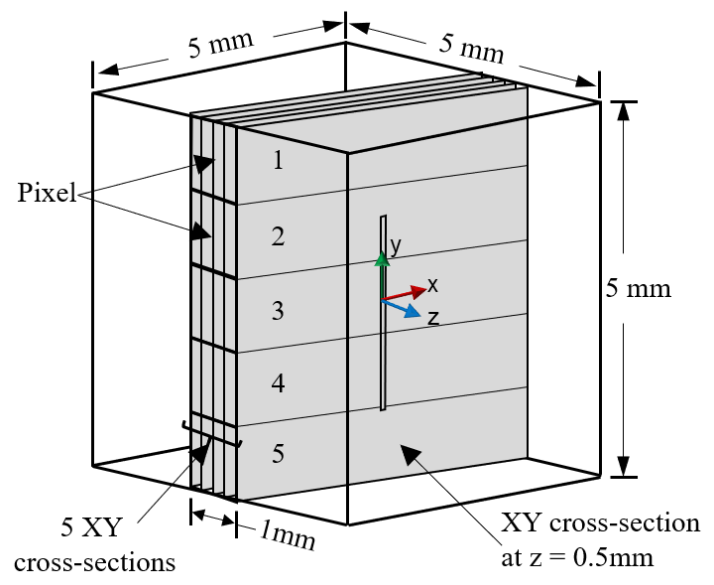

(a)

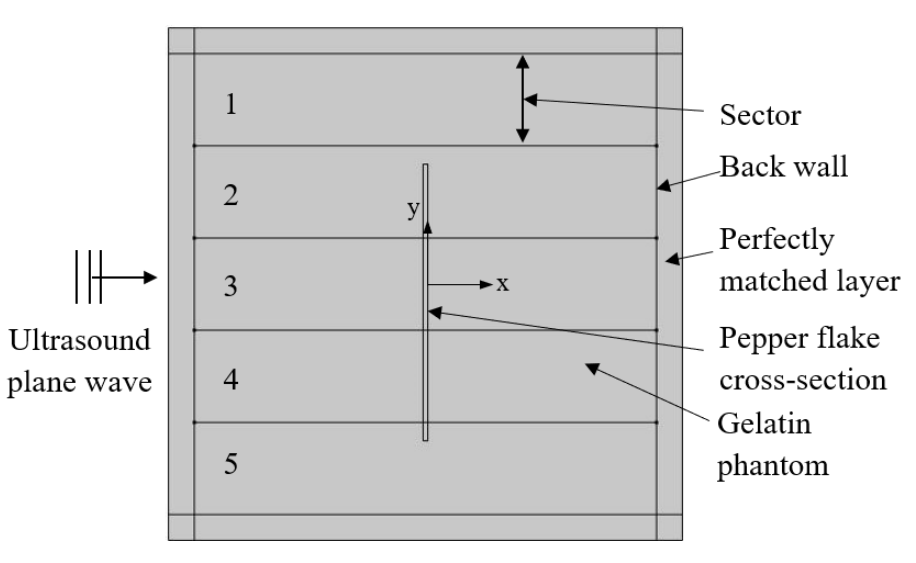

(b)

Figure 2. (a) Three-dimensional (3D) model with simplification details; (b) simplified 2D XY plane (at $Z=0$ ).

\subsection{Simulation Physics}

The soft pepper flake was considered the solid medium, and the surrounding gelatin phantom was considered the fluid medium. In the fluid medium, the acoustic wave propagation followed the Helmholtz equation shown in Equation (1) [24]. The equation provides the acoustic pressure distribution as a function of frequency inside the medium.

$$
\nabla^{2} p_{t}+k^{2} p_{t}=0
$$

The total pressure, $p_{t}$ is the summation of the background pressure field $\left(p_{b}\right)$ and backscatter pressure field $\left(p_{s}\right)$. In the model, the background pressure field was expressed as wave propagation in the $\hat{e_{x}}$ direction defined as $p_{b}=p_{0} e^{-k x} . k$ is the equivalent wave number defined for wave velocity $c_{c}$ in fluid and frequency $f$, and the attenuation coefficient $\alpha$ is expressed in Equation (2) [24].

$$
k=\frac{2 \pi f}{c_{c}}-i \ln (10) \frac{\alpha}{20}
$$

The pepper flake was modeled as a linear elastic solid. The acoustic pressure that was applied to the soft flake was very small $(\leq 1 \mathrm{~Pa})$. Therefore, the strain value of the solid flake was assumed to be too insignificant to follow a nonlinear stress-strain profile. Thus, a linear elastic approximation was considered. In the solid medium, the acoustic 
propagation was governed by Navier's equation shown in Equation (3) [24]. The equation provides solid displacement caused by the acoustic propagation at different frequencies.

$$
-(2 \pi f)^{2} \rho_{s} \boldsymbol{u}=\nabla . S+\boldsymbol{F} e^{i \phi}
$$

In this equation, $f$ is sound frequency, $S$ is Cauchy stress tensor, $\rho_{s}$ is solid density, $\phi$ is the phase component of the force, $\boldsymbol{F}$ is acting force on the solid, and $\boldsymbol{u}$ denotes the solid displacement field.

In the fluid-solid interface, in order to make sure that the sound pressure and structural acceleration got transferred between the fluid and solid, the following boundary conditions were used, which are shown in Equations (4) and (5) [24].

$$
\begin{gathered}
\boldsymbol{n} \cdot \frac{1}{\rho_{c}} \nabla p_{t}=-\boldsymbol{n} \cdot \boldsymbol{u}_{\boldsymbol{t} t} \\
\boldsymbol{F}=p_{t} \boldsymbol{n}
\end{gathered}
$$

$\boldsymbol{n}$ is the normal unit vector to the boundary, $\boldsymbol{u}_{t t}$ is structural acceleration, and $\boldsymbol{F}$ is an acting load on the solid.

In the fluid and solid domains, the material properties are listed in Table 1 [4,25].

Table 1. Material properties.

\begin{tabular}{cc}
\hline \multicolumn{3}{c}{ Gelatin Phantom (Fluid Region) } \\
\hline Density $\left(\rho_{1}\right)$ & $1067 \mathrm{~kg} / \mathrm{m}^{3}$ \\
Sound velocity & $1540 \mathrm{~m} / \mathrm{s}$ \\
Attenuation coefficient $(\alpha)$ & $8.05 \mathrm{~Np} / \mathrm{m}-\mathrm{MHz}$ \\
\hline \multicolumn{2}{c}{ Soft pepper flake (Solid domain) } \\
\hline Young's modulus & $1.152 \times 10^{7} \mathrm{~Pa}$ \\
Density & $608.1 \mathrm{~kg} / \mathrm{m}^{3}$ \\
Poisson's ratio & 0.295 \\
\hline
\end{tabular}

A free triangular mesh was created inside the fluid and solid domain. A mapped mesh was used in the perfectly matched layer. Mesh sensitivity analysis was conducted to achieve convergence in the simulation result. With decreasing mesh size, the scattered pressure was calculated for different frequency steps between 22 and $41 \mathrm{MHz}$. Convergence was achieved for the mesh element size of one-sixth of the wavelength. The simulation was conducted with a mesh of a maximum of 3,020,168 elements. A computational configuration of a Core i7-9700 processor with 16 GB RAM was used to conduct the simulation.

In the experimental study, the frequency bandwidth of the high-frequency transducer was $22-41 \mathrm{MHz}$ with a center frequency of $31.5 \mathrm{MHz}$ [22]. Therefore, a similar frequency range was used for the computational analysis. Furthermore, the transducer property was closely approximated in the input background pressure by multiplying it with a generalized Gaussian function of pressure distribution among the frequency range (Figure 3) [26]. This enabled us to apply frequency-dependent pressure similar to a transducer with frequency bandwidth. The frequency step size was selected as $100 \mathrm{kHz}$. The model was computed for the scattered pressure at each frequency step from 22 to $41 \mathrm{MHz}$, and then all the frequency responses were compiled into a frequency spectrum at the back wall. A peak counting algorithm was used to count the number of peaks and valleys of each spectrum. 


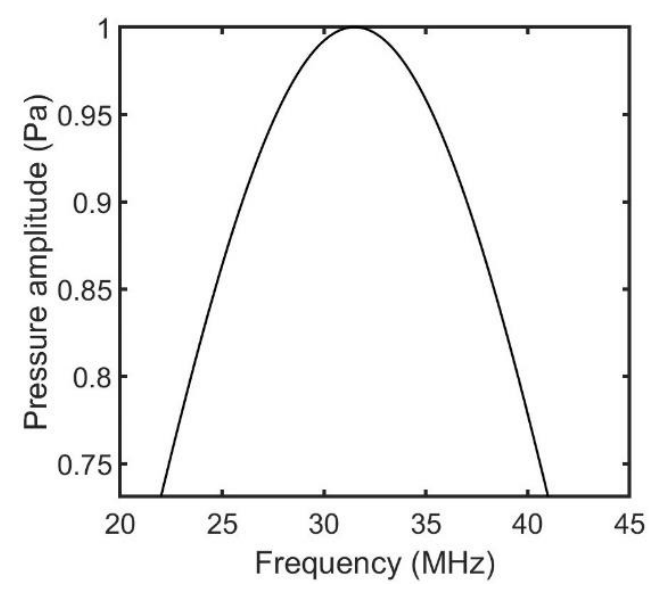

Figure 3. Incident background pressure distribution.

\subsection{Results and Discussion}

In the experimental work by Stromer et al., an intuitive threshold pressure magnitude was applied during the peak density calculation, which increased the image quality. The purpose of using the threshold value was to eliminate peaks with insignificant pressure magnitude. Similarly, in this study, a small threshold value of $0.02 \mathrm{~Pa}$ was applied during peak density calculation. The result shown in Figure 4 represents the peak density of the $X Y$ cross-section from the center of the Z-axis for all five sectors (Figure 2b). It was observed that peak density was higher in the sectors having a larger amount of pepper flake. Hence, Sector 1 had the lowest peak density, as there was no pepper flake in that sector. Sector 5 also showed a very low peak density instead of having a tiny portion of the pepper flake. During the image construction based on the peak density value, this kind of response (Sector 5) resulted in an image that did not visualize a portion of the structure (pepper flake) boundary. This issue was further discussed in the following sections.

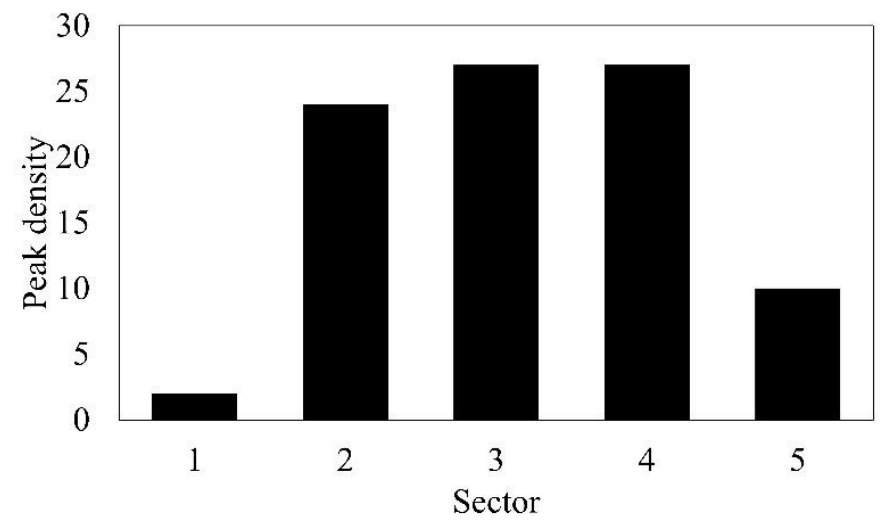

Figure 4. Peak density in all sectors at the center plane $(Z=0)$.

The received signal spectrum was also analyzed for the computational model described above. The purpose of this analysis was to explore the signal propagation in terms of the received signal pattern. In Figure 5, it was evident that in Sectors 1 and 5, the spectrums were similar to the Gaussian distribution of the incident pressure. This was because these sectors had very little or no amount of pepper flake, and the incident pressure field was almost uninterrupted by the pepper flake. In the case of Sectors 3 and 4 , all the incident waves went through the pepper flake domain, and the incident pressure spectrum was very much affected by the solid domain. 

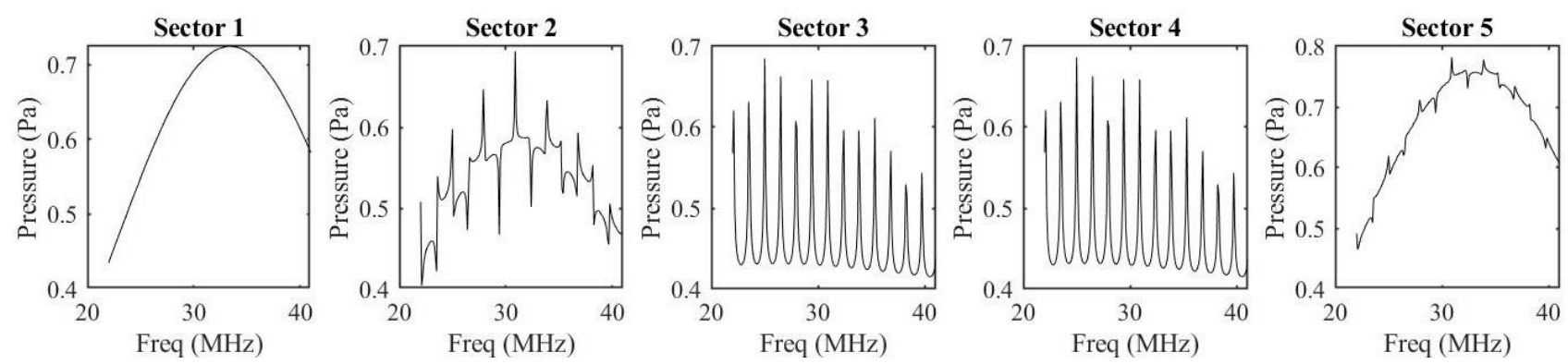

Figure 5. Frequency spectrum in different sectors at $Z=0$.

Figure 6 represents the C-scan image obtained from the computational model. From Figure $6 \mathrm{~b}$, it is evident that the pixel area containing the pepper flake had a higher value of peak density compared to the pixel areas having no pepper flake. The higher the peak density, the darker the pixel shade. The reconstructed image based on peak density value is shown in Figure 6c. Figure $6 \mathrm{c}$ delineates that almost all the pepper flake region was identified in the image except for the smaller region on the right side. This result was similar to the experimental results obtained by Stromer et al. [22].

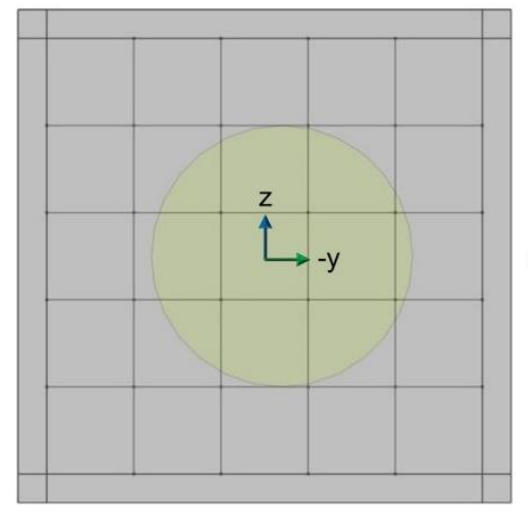

(a)

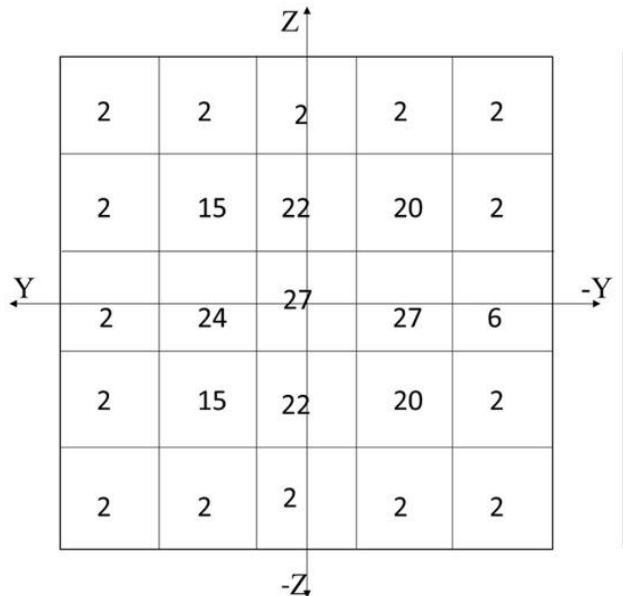

(b)

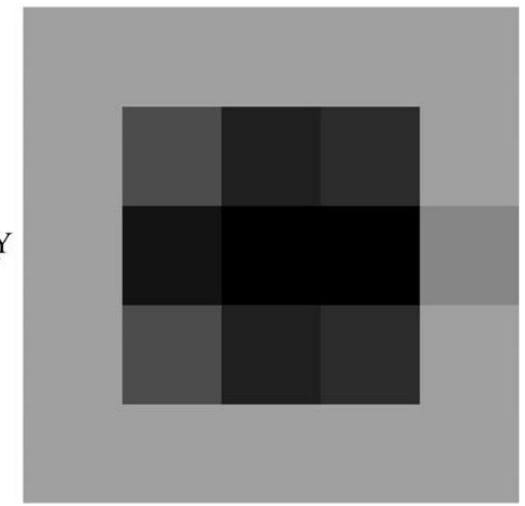

(c)

Figure 6. (a) Model geometry (YZ plane view); (b) peak density value of all pixels; (c) reconstructed image based on peak density.

To avoid the boundary detection issue and to image the object boundaries conclusively, the pixels could be selected in a way that the pepper flake sectors were completely covered by the flake. In that case, all the sectors containing pepper flake would return significantly higher peak density compared to the sectors that did not contain any pepper flake at all (Figure 4). Accordingly, pixel shades having pepper flakes would be more pronounced than the rest of the pixels.

\section{Structure Position Detection}

In C-scan imaging, although the presence of the structure and its properties can be detected, little information can be achieved regarding the structure's relative position along the sound propagation path. In the previous section, the effectiveness of the peak density was analyzed in the case of C-scan imaging. In this section, the feasibility of using the peak density will be discussed on detecting the position of the pepper flake in the direction of the gelatin phantom depth while conducting the C-scan imaging. 


\subsection{Model Description}

To conduct this study, another computational model was created where a cylindrical pepper flake was placed in the center of the phantom medium. In that way, Sectors 2-4 were completely covered by the rectangular flake cross-section, while Sectors 1 and 5 did not contain any flakes at all (Figure 7). Because the X-direction indicated the depth of the phantom, the rectangular cross-section was moved along the $X$-axis in both directions from the center of the phantom. Inside the $5 \mathrm{~mm}$ depth of the phantom, the flake was moved $0.5 \mathrm{~mm}$ each time, creating nine different positions for the flake inside the phantom, as shown in Figure 7. Because the flake was in a symmetrical position along the $Y$-axis, Sectors 2 and 4 resembled a similar type of sector. Therefore, the simulation was only conducted in Sectors 2 and 3 to detect the flake position. For each flake position, the peak density was calculated and then analyzed to observe any pattern in the peak density that could provide some information regarding its relative position.

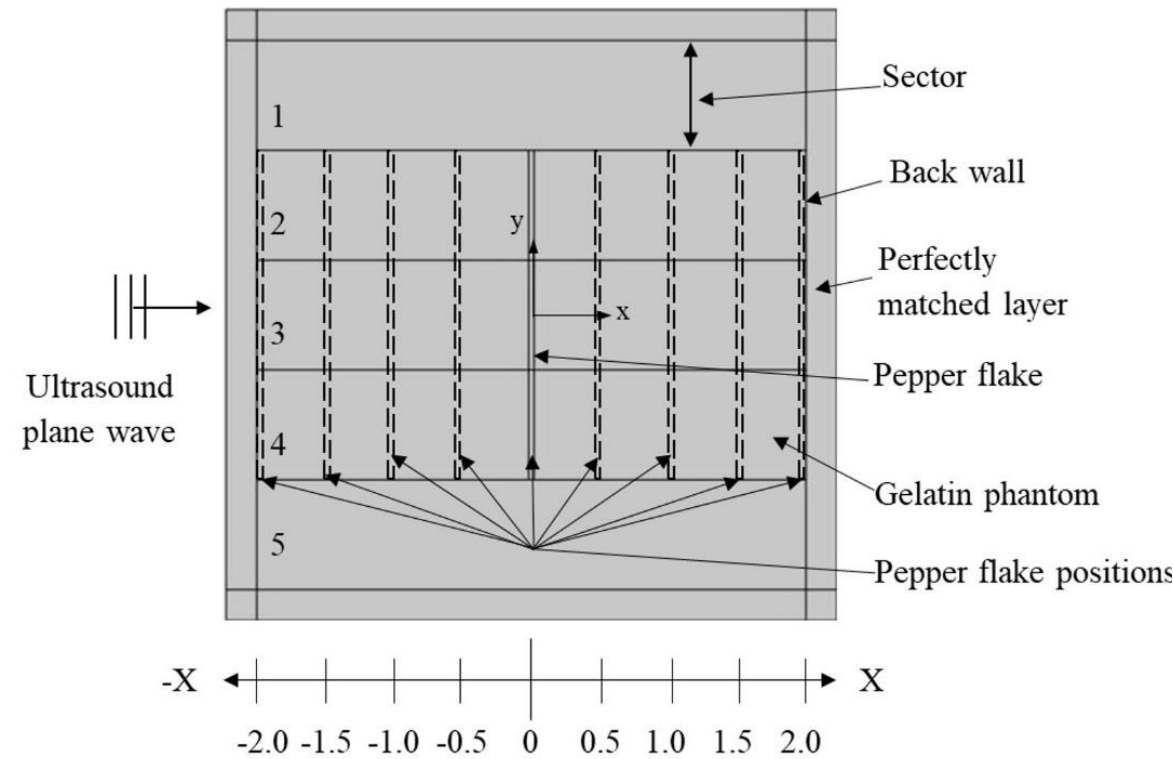

Figure 7. Pepper flake positions.

\subsection{Results and Discussion}

Figure 8a,b shows the peak density for the different positions of the flake at Sectors 2 and 3 , respectively. From both plots, it was observed that the peak density did not change with the flake position. Therefore, the frequency spectrums for both sectors at all positions were analyzed to find any additional correlation.

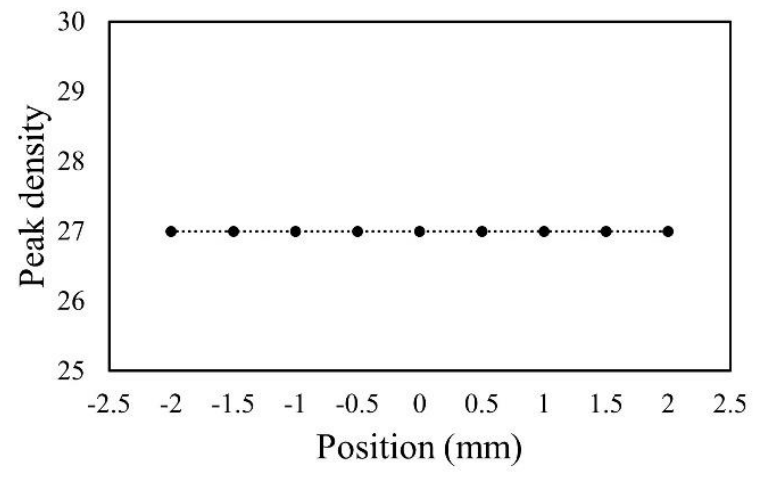

(a)

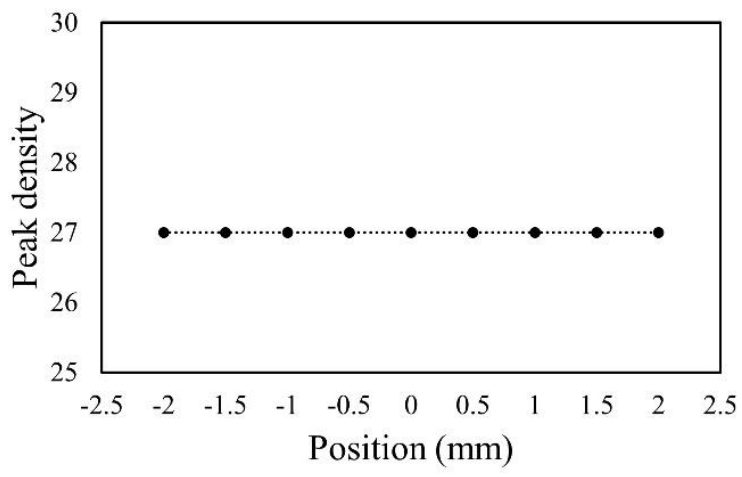

(b)

Figure 8. Peak density at all flake positions in (a) Sectors 2 and (b) 3. 
Figures 9 and 10 show the spectrums at all positions in Sectors 2 and 3, respectively. In both Sectors 2 and 3, the peaks and valleys showed a general pattern with pronounced peaks and valleys. This should be for the case of measuring the wave pressure at the back wall during its gradual compression and rarefaction stages at different frequencies. In both sectors, a similar frequency spectrum pattern was observed. However, a slight change in the pressure magnitude was noticed between peaks and valleys at different positions. Therefore, it was understood that, by studying the magnitude variation for those peaks and valleys, a conclusive correlation could be established between the flake position and magnitude variation. However, peak density was not able to depict the magnitude change in those peaks and valleys, as the increasing pressure value did not affect the peak density. Therefore, another parameter was developed to depict the magnitude variation while capturing the change in peak or valley magnitude. This parameter was named Mean Peak to Valley Distance (MPVD), as it measured the average magnitude difference between all adjacent peaks and valleys. To measure MPVD, the distance (pressure difference) was calculated from one peak to the next valley, and then from that valley to the next peak, and so on. All these distances were then averaged to achieve the final value. Therefore, the MPVD would exhibit a change, while the magnitude of the peaks and valleys changed in various locations.

(a)

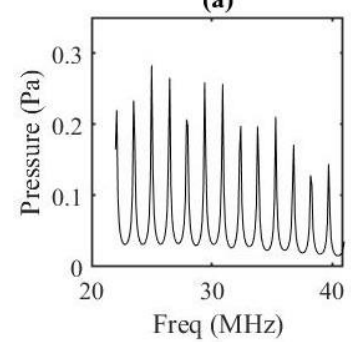

(f)

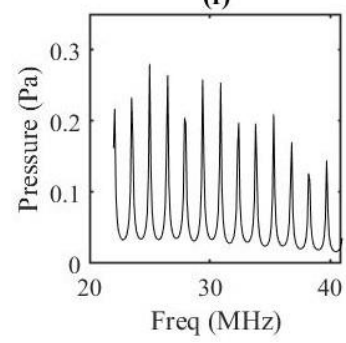

(b)

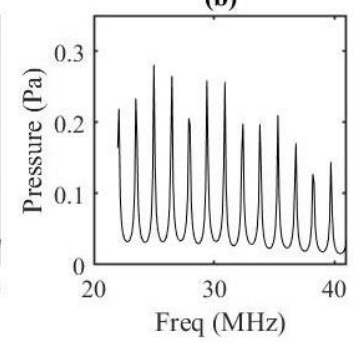

(g)

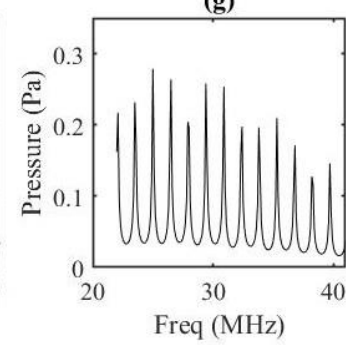

(c)

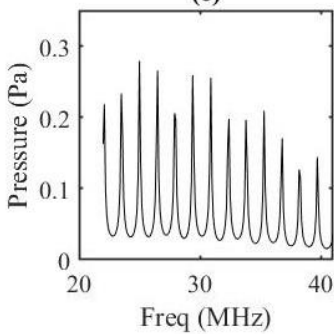

(h)

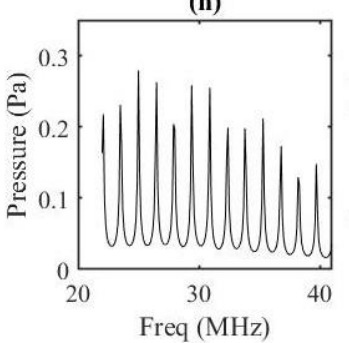

(d)

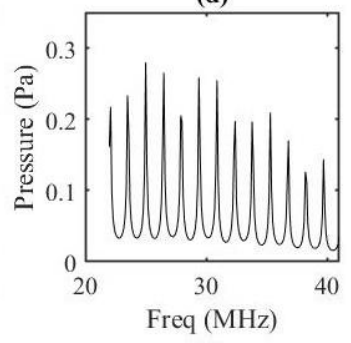

(i)

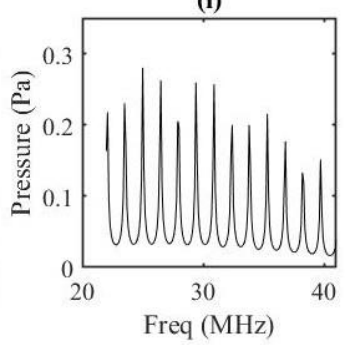

(e)

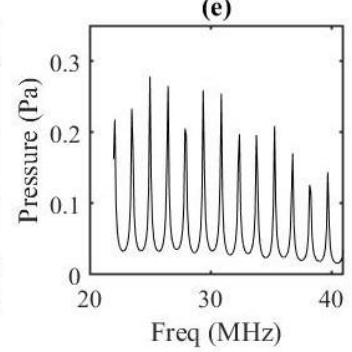

40

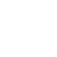

Figure 9. Frequency spectrum in Sector 2 for flake positioned at (a) $-2 \mathrm{~mm},(\mathbf{b})-1.5 \mathrm{~mm},(\mathbf{c})-1 \mathrm{~mm},(\mathbf{d})-0.5 \mathrm{~mm},(\mathbf{e}) 0 \mathrm{~mm}$, (f) $0.5 \mathrm{~mm},(\mathrm{~g}) 1 \mathrm{~mm},(\mathbf{h}) 1.5 \mathrm{~mm}$, and (i) $2 \mathrm{~mm}$.

Figure 11a,b shows the MPVD results for Sectors 2 and 3 at all the locations of the pepper flake inside the phantom. In both sectors, the MPVD value was found to gradually increase from the central location while following a second-degree polynomial trendline. Furthermore, the increasing rate of the MPVD value was higher while the flake got closer to the back wall (0 to $2 \mathrm{~mm}$ ) compared to the flake getting closer to the ultrasound source ( 0 to $-2 \mathrm{~mm}$ ). The primary reason behind the polynomial trend should be the flake's various positions where it interacted with the compression/rarefaction phases of the sound wave at those positions. However, while the flake moved towards the back wall or bottom of the phantom, the scattered wave from the flake started attenuating less because of the decreasing travel path. Thus, scattered pressure started to increase at the back wall. The increasing pressure contributed to the MPVD value and affected the polynomial trend. Therefore, from -2 to $0 \mathrm{~mm}$, the difference between MPVD values (negative slope) decreased, while from 0 to $2 \mathrm{~mm}$, the positive slope increased. One important factor is that the difference between MPVD values was very small compared to the original spectrum's pressure magnitude. Therefore, to perform this analysis at the experimental level, the 
frequency spectrums need to be normalized by reference signal in order to avoid the noise effect during the MPVD calculation [27].

(a)

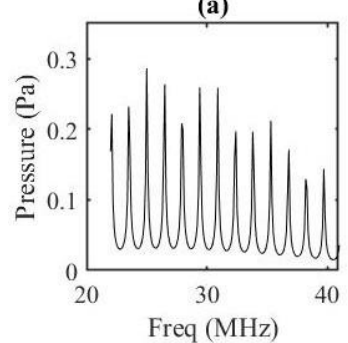

(f)

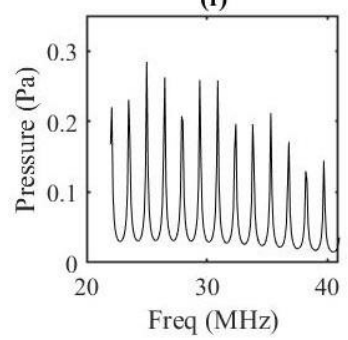

(b)

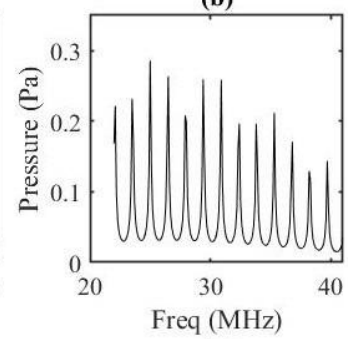

(g)

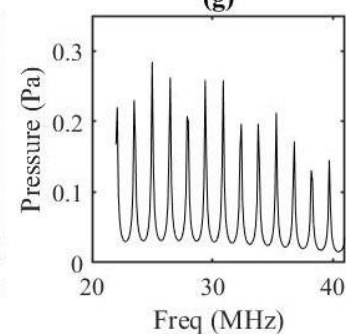

(c)

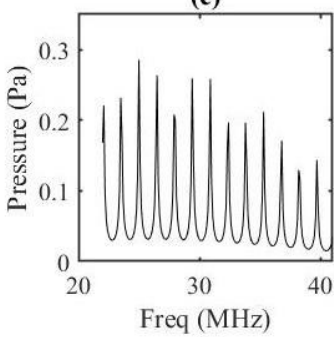

(h)

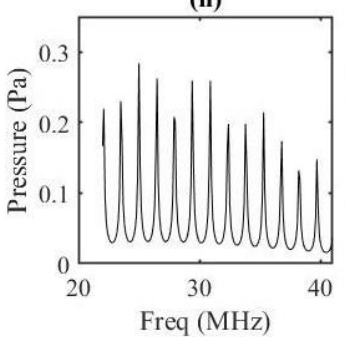

(d)

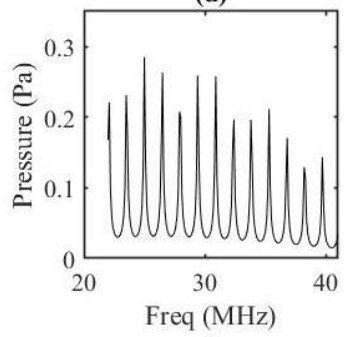

(i)

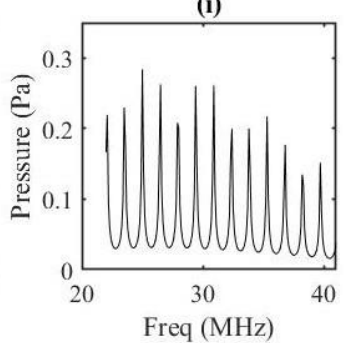

(e)
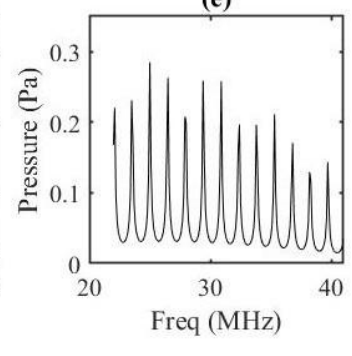

Figure 10. Frequency spectrum in Sector 3 for flake positioned at (a) $-2 \mathrm{~mm},(\mathbf{b})-1.5 \mathrm{~mm},(\mathbf{c})-1 \mathrm{~mm},(\mathbf{d})-0.5 \mathrm{~mm},(\mathbf{e}) 0$ $\mathrm{mm},(\mathbf{f}) 0.5 \mathrm{~mm},(\mathrm{~g}) 1 \mathrm{~mm}$, (h) $1.5 \mathrm{~mm}$, and (i) $2 \mathrm{~mm}$.

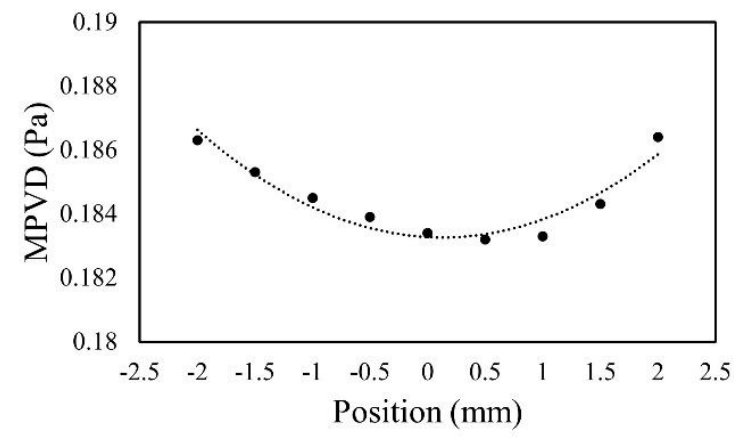

(a)

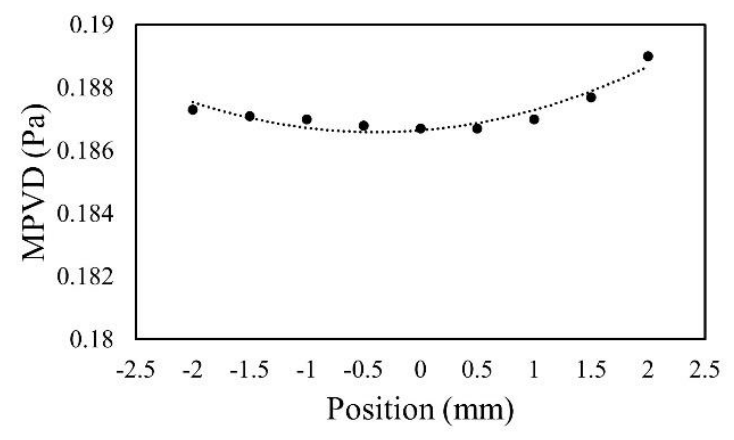

(b)

Figure 11. MPVD value at all flake positions in (a) Sectors 2 and (b) 3.

\section{Discussion}

In this study, the feasibility of the magnitude variation of the transmitted ultrasound signal in the frequency domain was analyzed for C-scan imaging. The magnitude variation was expressed in terms of peak density and MPVD. In a previous experimental study by Stromer et al., peak density was found as a promising nonconventional parameter for C-scan imaging [22]. The study documented some inefficiencies for peak density to detect the boundary of the structure in the case of high-frequency analysis. Therefore, in this study, first, the experimental result from Stromer et al. was imitated in the computational C-scan imaging model, which had a similar issue of boundary detection [22]. From the result, it was observed that peak density successfully generated a C-scan image of a thin pepper flake. Although peak density could not conclusively detect the flake's boundary in the pixel areas with a tiny amount of pepper flake, the issue could be avoided by proper pixel area selection. Furthermore, if a smaller pixel size can be achieved for the imaging through a smaller transducer step size, most of the pepper flakes will be automatically placed fully inside the pixel area, resulting in a better image. Even after that, there is a chance of the flake being positioned partially. However, it would be a negligible amount, and thus the boundary detection would be much better. The smaller pixel can also be achieved 
experimentally by decreasing the ultrasound beam width. We already know that a smaller beam width is better for the lateral resolution for the conventional ultrasound parameter in $\mathrm{C}$-scan imaging. Therefore, it is evident that with an unconventional parameter like peak density, a smaller beam width would increase the resolution of the image.

In C-scan imaging, minimal information can be gathered about the structure position along with the depth of the sample. Therefore, studies were conducted by utilizing the magnitude variation to see the feasibility of achieving any information about the structure location. The cylindrical pepper flake was moved along the depth of the phantom for this study, and magnitude variation was evaluated in terms of peak density for all locations. It was observed that peak density was not able to capture the change in the spectrum properly, as peaks and valleys were changing their magnitude level, resulting in similar peak densities for different positions. Thus, peak density could not establish any relationship with structure position. Therefore, the MPVD parameter was used to express the magnitude variation. MPVD perfectly captured the magnitude change of peaks and valleys. With gradually positioning the flake from the middle to the bottom of the phantom as well as to the transducer, the MPVD value showed a strong increasing trend. Therefore, the MPVD value can give us information about the structure location while creating C-scan images. To have more accurate information, only the significant peak and valley magnitude can be observed. It is expected that with different shapes of the structures, significant peaks and valleys will be created at different frequency steps. It can also create a different pattern of the signal. Thus, by analyzing the signal pattern more information can be gathered regarding the shape of the structure without imaging the sample.

In the medical sector, this C-scan imaging technique can be used to image tissue heterogeneity. To detect cancerous margins during breast-conserving therapy (BCT), it can be used to identify positive/negative margins instantly. The main challenge of using high-frequency ultrasound in C-scan imaging would be its increasing attenuation rate, which limits this application to a low thickness level. In recent years, research has been conducted to evaluate quantitative ultrasound parameters in photoacoustic measurement and imaging $[28,29]$. Therefore, future research can be performed to evaluate the sensitivity of peak density to optical absorbance. With a promising result, this technique can be merged with photoacoustic C-scan imaging for much better imaging performance.

\section{Conclusions}

Computational modeling of ultrasound C-scan imaging was developed to analyze the feasibility of the peak density parameter of the transmitted frequency spectrum. A previous experimental study was imitated in the computational model for the validation of the analysis. The thin structure was successfully imaged through the computational study. With proper pixel area selection or smaller pixel size, a better boundary detection can be achieved in C-scan imaging while using the ultrasound peak density parameter. Peak density was not effective in giving information regarding the relative position of the structure along with the sample depth. Therefore, the magnitude variation of the signal was analyzed with the MPVD parameter instead of peak density to detect structure position. With the increasing depth of the pepper flake inside the gelatin phantom, the MPVD value changed while following a polynomial function. Therefore, in pitch-catch analysis, magnitude variation of the frequency spectrum is an effective tool for C-scan imaging as well as for extracting additional information about structure position. Further research is ongoing to evaluate the feasibility of this C-scan imaging technique to image various histological features of cancerous tissue. In the future, the feasibility of photoacoustic C-scan imaging will also be explored while using a similar parameter.

Author Contributions: Conceptualization, K.P. and L.L.; methodology, L.L., K.P. and J.S.; software, K.P.; validation, K.P. and J.S.; formal analysis, L.L. and K.P.; investigation, L.L. and K.P.; resources, L.L. and J.R; data curation, K.P.; writing-original draft preparation, K.P.; writing-review and editing, K.P., J.S., S.R., B.A.P., L.L. and J.R.; visualization, S.R., B.A.P. and L.L.; supervision, L.L. and J.R.; 
project administration, L.L. and J.R.; funding acquisition, L.L. and J.R. All authors have read and agreed to the published version of the manuscript.

Funding: This research received no external funding.

Institutional Review Board Statement: Not applicable.

Informed Consent Statement: Not applicable.

Data Availability Statement: Not applicable.

Conflicts of Interest: The authors declare no conflict of interest.

\section{References}

1. Mercado, K.P.E. Developing High-Frequency Quantitative Ultrasound Techniques to Characterize Three-Dimensional Engineered Tissues. Ph.D. Thesis, University of Rochester, Rochester, NY, USA, 2015.

2. Nam, S.Y.; Ricles, L.M.; Suggs, L.J.; Emelianov, S.Y. Imaging Strategies for Tissue Engineering Applications. Tissue Eng. Part B Rev. 2015, 21, 88-102. [CrossRef]

3. Szabo, T.L. Diagnostic Ultrasound Imaging: Inside Out; Elsevier: Amsterdam, The Netherlands, 2004.

4. Kremkau, F.W. Sonography Principles and Instruments; Elsevier: Amsterdam, The Netherlands, 2014.

5. Versaci, M.; Morabito, F.C. Image Edge Detection: A New Approach Based on Fuzzy Entropy and Fuzzy Divergence. Int. J. Fuzzy Syst. 2021. [CrossRef]

6. Madhukumar, S.; Santhiyakumari, N. Evaluation of k-Means and fuzzy C-means segmentation on MR images of brain. Egypt. J. Radiol. Nucl. Med. 2015, 46, 475-479. [CrossRef]

7. Gordon, G.; Canumalla, S.; Tittmann, B. Ultrasonic C-scan imaging for material characterization. Ultrasonics 1993, 31, 373-380. [CrossRef]

8. Kundu, T.; Ehsani, M.; Maslov, K.; Guo, D. C-scan and L-scan generated images of the concrete/GFRP composite interface. NDT E Int. 1999, 32, 61-69. [CrossRef]

9. Thornton, M.; Han, L.; Shergold, M. Progress in NDT of resistance spot welding of aluminium using ultrasonic C-scan. NDT E Int. 2012, 48, 30-38. [CrossRef]

10. Růžek, R.; Lohonka, R.; Jironč, J. Ultrasonic C-Scan and shearography NDI techniques evaluation of impact defects identification. NDT E Int. 2006, 39, 132-142. [CrossRef]

11. Restori, M.; Wright, J.E. C-scan ultrasonography in orbital diagnosis. Br. J. Ophthalmol. 1977, 61, 735-740. [CrossRef]

12. Hasiotis, T.; Badogiannis, E.; Tsouvalis, N.G. Application of Ultrasonic C-Scan Techniques for Tracing Defects in Laminated Composite Materials. J. Mech. Eng. 2011, 2011, 192-203. [CrossRef]

13. Ye, S.; Harasiewicz, K.; Pavlin, C.; Foster, F. Ultrasound characterization of normal ocular tissue in the frequency range from 50 $\mathrm{MHz}$ to $100 \mathrm{MHz}$. IEEE Trans. Ultrason. Ferroelectr. Freq. Control 1995, 42, 8-14. [CrossRef]

14. Jin, H.; Liu, S.; Zhang, R.; Liu, S.; Zheng, Y. Frequency Domain Based Virtual Detector for Heterogeneous Media in Photoacoustic Imaging. IEEE Trans. Comput. Imaging 2020, 6, 569-578. [CrossRef]

15. Imielińska, K.; Castaings, M.; Wojtyra, R.; Haras, J.; Le Clezio, E.; Hosten, B. Air-coupled ultrasonic C-scan technique in impact response testing of carbon fibre and hybrid: Glass, carbon and Kevlar/epoxy composites. J. Mater. Process. Technol. 2004, 157-158, 513-522. [CrossRef]

16. Garcia, J. A 3-dimensional ultrasound C-scan imaging technique for optic nerve measurements. Ophthalmology 2004, 111, 1238-1243. [CrossRef] [PubMed]

17. Ghoshal, G.; Oelze, M.L.; O’Brien, W.D., Jr. Quantitative Ultrasound History and Successes. In Quantitative Ultrasound in Soft Tissues; Springer: Dordrecht, The Netherlands, 2013; pp. 21-42.

18. Gudur, M.; Rao, R.R.; Hsiao, Y.-S.; Peterson, A.W.; Deng, C.X.; Stegemann, J.P. Noninvasive, Quantitative, Spatiotemporal Characterization of Mineralization in Three-Dimensional Collagen Hydrogels Using High-Resolution Spectral Ultrasound Imaging. Tissue Eng. Part C Methods 2012, 18, 935-946. [CrossRef] [PubMed]

19. Lizzi, F.L.; Ostromogilsky, M.; Feleppa, E.J.; Rorke, M.C.; Yaremko, M.M. Relationship of ultrasonic spectral parameters to features of tissue microstructure. IEEE Trans. Ultrason. Ferroelectr. Freq. Control 1987, 34, 319-329. [CrossRef] [PubMed]

20. Sethuraman, S.; Amirian, J.H.; Litovsky, S.H.; Smalling, R.W.; Emelianov, S.Y. Ex vivo Characterization of Atherosclerosis using Intravascular Photoacoustic Imaging. Opt. Express 2007, 15, 16657-16666. [CrossRef]

21. Doyle, T.E.; Factor, R.E.; Ellefson, C.L.; Sorensen, K.M.; Ambrose, B.J.; Goodrich, J.B.; Hart, V.P.; Jensen, S.C.; Patel, H.; Neumayer, L.A. High-frequency ultrasound for intraoperative margin assessments in breast conservation surgery: A feasibility study. BMC Cancer 2011, 11, 444. [CrossRef]

22. Stromer, J.; Ladani, L. Investigating ultrasound imaging in the frequency domain for tissue characterisation. Nondestruct. Test. Eval. 2015, 31, 209-218. [CrossRef]

23. Ladani, L.; Paul, K.; Stromer, J. High-Frequency Ultrasound Analysis in Both Experimental and Computation Level to Understand the Microstructural Change in Soft Tissues. In Minerals, Metals and Materials Series; Springer International Publishing: Berlin/Heidelberg, Germany, 2019; pp. 87-97. 
24. Paul, K.; Ladani, L. Relationship between peak density and acoustic scattering in high-frequency ultrasound wave propagation. SN Appl. Sci. 2020, 2, 1-12. [CrossRef]

25. Nam, J.; Byun, J.; Kim, T.; Kim, M.; Kim, D. Measurement of Mechanical and Physical Properties of Pepper for Particle Behavior Analysis. J. Biosyst. Eng. 2018, 43, 173-184.

26. Delrue, S.; Abeele, K.V.D.; Blomme, E.; Deveugele, J.; Lust, P.; Matar, O.B. Two-dimensional simulation of the single-sided air-coupled ultrasonic pitch-catch technique for non-destructive testing. Ultrasonics 2010, 50, 188-196. [CrossRef] [PubMed]

27. Stromer, J.; Ladani, L. Examination of a spectral-based ultrasonic analysis method for materials characterization and evaluation. Biomed. Signal Process. Control 2018, 40, 454-461. [CrossRef]

28. Lashkari, B.; Yang, L.; Mandelis, A. The application of backscattered ultrasound and photoacoustic signals for assessment of bone collagen and mineral contents. Quant. Imaging Med. Surg. 2015, 5, 46-56. [PubMed]

29. Xi, L.; Zhou, L.; Jiang, H. C-scan photoacoustic microscopy for invivo imaging of Drosophila pupae. Appl. Phys. Lett. 2012, 101, 013702. [CrossRef] 